\title{
The effects of stimulus pulse rise-decay time and duration on lingual vibrotactile thresholds
}

\author{
DONALD FUCCI, DANIEL HARRIS, and LINDA PETROSINO \\ School of Hearing and Speech Sciences, Ohio University, Athens, Ohio 45701
}

\begin{abstract}
The present study consisted of two experimental phases. In Experiment 1, the effects on lingual vibrotactile threshold of four different stimulus pulse rise-decay times were investigated for 10 normal adult subjects. The results showed no statistically significant differences between thresholds obtained with the use of the four rise-decay times. In Experiment 2, the effects on lingual vibrotactile threshold of four different stimulus pulse durations were investigated for 10 normal adult subjects. A statistically significant difference was noted between thresholds obtained with stimulus pulse durations of land $50 \mathrm{msec}$.
\end{abstract}

A number of investigations have been performed to determine significant stimulus parameters for measurement of lingual vibrotactile thresholds. Telage, Fucci, and Arnst (1972) found that maximum sensitivity occurred within a frequency range of $250-400 \mathrm{~Hz}$. Fucci, Arnst, Telage, and McCaffrey (1973) demonstrated that the parameters of pulsed vs. continuous stimulation affected thresholds. Fucci et al. found that lowest values were obtained using an ascending pulsed signal. Telage and Warren (1977) demonstrated that thresholds decreased when contactor size was increased. The greatest decreases were noted for the two largest contactors of the four sizes employed.

Telage, Fucci, and Blackmon (1976) identified the significance of stimulus pulse duration as a parameter for obtaining lingual vibrotactile thresholds. Measurements were obtained using a $250-\mathrm{Hz}$ signal with burst durations of $50,100,200$, and $400 \mathrm{msec}$. For each burst duration, a rise-decay time of $25 \mathrm{msec}$ was used. The results demonstrated a decrease in threshold corresponding to each increase in duration.

The purpose of the present pilot study is to further investigate the effects of stimulus pulse rise-decay time and duration on lingual vibrotactile thresholds by varying the rise-decay times and by extending the lower end of the range of durations used by Telage et al. (1976).

This investigation was performed in two experimental phases. In Experiment 1, four rise-decay times are used with a $250-\mathrm{Hz}$ pulsed stimulus. Previous experiments have not investigated the effects of varying rise-decay times on thresholds. In Experiment 2, four pulse durations are used with a $250-\mathrm{Hz}$ pulsed stimulus.

\section{EXPERIMENT 1}

\section{Method}

Subjects. Ten young adults reporting no history of speech and/or hearing disorders served as subjects for this experiment. None of the subjects was experienced in lingual vibrotactile threshold technique. The subjects ranged in age from 18 to 20 years, with a mean age of 18.8 years.

Apparatus. The vibrotactile instrumentation consisted of a stimulus unit and a measurement unit. The stimulus unit included a sine-wave generator, an audio amplifier, a rise-decay gate, two universal timers, a 2-dB step-variable attenuator, a power amplifier, and an electromagnetic minivibrator. The pulsed vibratory signal generated had a 50\% duty cycle (on $.5 \mathrm{sec}$ and off $.5 \mathrm{sec}$ ). The measurement unit consisted of an accelerometer, a cathode follower, a microphone amplifier, and a dual beam storage oscilliscope. The equipment used in this study has been described in previous publications, the most recent being an article on modification of the instrumentation (Fucci, Petrosino, Wallace, \& Small, 1982).

Procedure. Each subject was seated in an adjustable chair and asked to place his tongue up against the bottom of a plastic disk. A hole in the disk provided access of the probe to the anterior midline section of the tongue. To maintain constant pressure of the vibrator on the tongue for all subjects, the probe was lowered until a voltmeter recorded contact. The probe was then lowered $1 \mathrm{~mm}$ into the tongue surface from the point of recorded contact.

Subjects received training to detect the presence of a $250-\mathrm{Hz}$ pulsed signal on the tongue. The signal generated had a $100-\mathrm{msec}$ rise-decay time. Training was considered to be complete when the subject could detect three successive stimuli within a $10-\mathrm{mV}$ range. An ascending psychophysical method of limits was used during training and subsequent measurements.

Subsequent to adequate training, lingual vibrotactile thresholds were obtained using a $250-\mathrm{Hz}$ pulsed signal. The signal pulses had a duration of $500 \mathrm{msec}$ for all conditions. The risedecay times of the signal were varied to include $1,25,50$, and $100 \mathrm{msec}$. For each stimulus condition, six millivolt readings were taken for each subject's threshold. The first three read-

Table 1

Mean Thresholds (in Microns) and Standard Deviations as a Function of the Stimulus Pulse Rise-Decay Time

\begin{tabular}{ccc}
\hline $\begin{array}{l}\text { Rise-Decay Time } \\
\text { (in Milliseconds) }\end{array}$ & Mean & SD \\
\hline 1 & 1.64 & .55 \\
25 & 1.87 & .61 \\
50 & 1.68 & .63 \\
100 & 1.79 & .79 \\
\hline
\end{tabular}


ings were discarded, and the mean of the remaining three was accepted as threshold. All thresholds were converted to peak displacement in microns. Arithmetic means and standard deviations calculated over the performance of the group for the four stimulus rise-decay times are presented in Table 1 .

\section{Results}

Table 1 shows the mean displacement in microns and standard deviations for the thresholds obtained over the four stimulus rise-decay conditions. It can be seen that means and standard deviations are somewhat similar across all four conditions. A repeated-measures analysis of variance was performed to determine if there were statistically significant differences among the means (Bruning \& Kintz, 1977). The F ratio obtained was not significant at the .05 level $[F(3,27)=1.02, p=.40]$. It appears from the results that none of the four stimulus rise-decay times differed significantly in their effect on lingual vibrotactile thresholds.

\section{EXPERIMENT 2}

\section{Method}

Subjects. Ten young adults who did not participate in Experiment 1 served as subjects for Experiment 2. They reported no history of speech and/or hearing disorders. None of the subjects was experienced in lingual vibrotactile threshold technique. The subjects ranged in age from 18 to 20 years, with a mean age of 19.0 years.

Apparatus. The instrumentation in Experiment 2 was the same as that used in Experiment 1.

Procedure. Each subject was positioned and trained to detect threshold on the tongue in the same manner as the subjects in Experiment 1.

Subsequent to adequate training, lingual vibrotactile thresholds for a $250-\mathrm{Hz}$ pulsed signal were obtained at pulse durations of $1,25,50$, and $125 \mathrm{msec}$. A rise-decay time of $1 \mathrm{msec}$ was used for all test conditions. Six millivolt readings were taken for each subject's threshold. The first three readings were discarded, and the mean of the remaining three was accepted as threshold. All thresholds were converted to peak displacement in microns. Arithmetic means and standard deviations calculated over the performance of the group for the four stimulus duration times are presented in Table 2.

\section{Results}

Table 2 shows the mean displacement in microns and standard deviations for the thresholds obtained over the four stimulus duration conditions. A repeated-measures analysis of variance was performed to determine if there were statistically significant differences among the means. The $F$ ratio obtained was significant at the .05

Table 2

Mean Thresholds (in Microns) and Standard Deviations as a Function of the Stimulus Pulse Duration (in Milliseconds)

\begin{tabular}{ccc}
\hline Duration & Mean & SD \\
\hline 1 & .23 & .07 \\
25 & .32 & .10 \\
50 & .33 & .13 \\
125 & .27 & .07 \\
\hline
\end{tabular}

level $[F(3,27)=3.33, p=.02]$ (Bruning \& Kintz, 1977). A Tukey multiple-comparison test was then performed to determine which means were significantly different. A significant difference was found between the threshold mean for $1 \mathrm{msec}$ duration and the threshold mean for $50 \mathrm{msec}$ duration. No other significant differences were found.

\section{DISCUSSION}

Statistical analysis indicated that the rise-decay times investigated do not appear to significantly affect lingual vibrotactile thresholds. The increase in stimulus pulse durations from 1 to $50 \mathrm{msec}$ appeared to significantly affect thresholds, whereas nonsignificant differences were observed between pulse durations of 1,25 , and 125 msec. Telage et al. (1976) have previously established that as pulse duration is increased, the intensity required to elicit threshold decreases. They found that thresholds decreased more sharply from 50 to $200 \mathrm{msec}$ than from 200 to $400 \mathrm{msec}$. They state that the more sharply sloping function from 50 to $200 \mathrm{msec}$ is indicative of the temporal summation effect related to pulse duration. Temporal summation as a sensory process is defined by Verrillo (1965) as the functional relationship that exists between some temporal pattern of a stimulus and the intensity level required to elicit a response at threshold levels. Telage et al. (1976) noted that extension of the slope beyond $200 \mathrm{msec}$ shows diminished evidence of temporal summation. An asymptote seems to be approached around 400 msec.

In the current study, the significant difference found between the threshold means for 1 and $50 \mathrm{msec}$ supports the data of Telage et al. (1976). A threshold mean reduction of .10 microns was observed between conditions of 1 - and $50-\mathrm{msec}$ stimulus pulse durations. Data reported by Verrillo (1965) show a comparable vibrotactile threshold shift for nonoral structures. In his study, the fleshy pad over the first metacarpal of the right hand was stimulated with pulsed signals of 100,250 , and $500 \mathrm{~Hz}$. Pulse durations were 10, 20,30, 50,70,100,300, and $1,100 \mathrm{msec}$. A rise-decay time of $5 \mathrm{msec}$ was used for all conditions. Contactor sizes used were $.02, .05, .08$, and $2.9 \mathrm{~cm}^{2}$. Decreases in thresholds were observed corresponding to increases in pulse durations from 20 to $300 \mathrm{msec}$. The greatest decreases in thresholds were observed with use of the largest contactor over the three experimental frequencies. Verrillo suggests that the observed decreases in thresholds are a result of the effect of temporal summation related to pulse duration.

In conclusion, it can be stated that the rise-decay times used in this study appeared to have no significant effect on lingual vibrotactile thresholds obtained from the anterior midline surface of the tongue. The increase in stimulus pulse duration from 1 to $50 \mathrm{msec}$ appeared to have a significant effect on thresholds The fact that thresholds appear to be affected by some alterations in pulse duration suggests that temporal summation may be an area of interest for continued investigation in oral vibrotactile research.

\section{REFERENCES}

Brunina, J., \& Kintz, B. Computational handbook of statistics. Glenview, Ill: Scott, Foresman, 1977.

Fucci, D., Arnst, D., Telage, K., \& McCaffrey, P. Measurement of lingual vibrotactile sensitivity using pulsed and continuous stimulation. Bulletin of the Psychonomic Society, 1973, 2, 85-86.

Fucci, D., Petrosino, L., Wallace, L., \& Small, L. Modification of instrumentation for research on lingual vibrotactile sensitivity: Elimination of the tongue clamping procedure. Review of Scientific Instrumentation, 1982, 53, 1294-1296. 
Telage, K., Fucci, D., \& Arnst, D. Normative study of oral vibrotactile sensitivity. Perceptual and Motor Skills, 1972, 35, 671-676.

Telage, K., Fucci, D., \& Blackmon, R. Temporal summation effects on lingual vibrotactile thresholds. Perceptual and Motor Skills, 1976, 42, 859-864.

Telage, K., \& Warren, J. Spatial summation effects on lingual vibrotactile thresholds. Perceptual and Motor Skills, 1977, 44, 1179-1185.

VERRILLO, R. Temporal summation in vibrotactile sensitivity. Journal of the Acoustical Society of America, 1965, 37, 843-846.

(Received for publication December 21, 1982.) 